ESTUDIO COMPARATIVO DE NIVELES DE ESTRÉS ENTRE ÁREAS FUNCIONALES DE EMPRESAS FAMILIARES. EL PARADIGMA DE ÁREAS DURAS Y BLANDAS.

\title{
ESTUDIO COMPARATIVO DE NIVELES DE ESTRÉS ENTRE ÁREAS FUNCIONALES DE EMPRESAS FAMILIARES. EL PARADIGMA DE ÁREAS DURAS Y BLANDAS
}

\section{COMPARATIVE STUDY OF STRESS LEVELS AMONG FUNCTIONAL AREAS OF FAMILY BUSINESSES. THE PARADIGM OF HARD AND SOFT AREAS}

José Vili Martínez González*, Vili Aldebarán Martínez García**

* Doctor en Administración. Ajedrez Consultores. Secretario General del COLPARMEX. Ecatepec, Estado de México. ORCID: http://orcid.org/0000-0002-0978-5778.

** Joven Investigador de Ajedrez Consultores. Ecatepec, Estado de México. ORCID: http://orcid.org/00000002-3901-0121.

Dirección para recibir correspondencia: ajedrezconsultores@hotmail.com 


\title{
Original
}

ESTUDIO COMPARATIVO DE NIVELES DE ESTRÉS ENTRE ÁREAS FUNCIONALES DE EMPRESAS FAMILIARES. EL PARADIGMA DE ÁREAS DURAS Y BLANDAS.

\section{RESUMEN}

La Organización Internacional del Trabajo (OIT), identifica al estrés como el primer signo de una respuesta a un daño físico y emocional, el cual podría suponer pérdidas de entre el $0.5 \%$ y el 3.5\% del Producto Interno Bruto (PIB) de cada país. Existe el paradigma dentro de las empresas referente a, que áreas como producción o finanzas son más propensas a presentarlo, que sus contrapartes de recursos humanos o mercadotecnia.

OBJETIVO: Identificar si existe diferencia en los niveles de estrés entre dichas áreas en las unidades de estudio seleccionadas.

MATERIAL Y MÉTODO: Se realizó una investigación de tipo descriptiva, transeccional, utilizando Aldebarán-Test para identificar el nivel de estrés laboral, utilizando una muestra de sujeto tipo con un total de 35 empresas familiares medianas ubicadas en el Valle de México, considerando a los gerentes y jefes de las áreas de producción, finanzas, recursos humanos y mercadotecnia, integrándose por un total de 280 participantes.

RESULTADOS: En las cuatro áreas seleccionadas, los niveles de estrés resultaron ser muy similares y ser los factores organizacionales los que presentaron una mayor incidencia.

CONCLUSIONES: Los hallazgos obtenidos brindan información para coadyuvar, tanto a identificar factores concomitantes en lo relativo a los niveles de estrés como al diseño de estrategias específicas para su control.

PALABRAS CLAVE: Estrés laboral. Empresas familiares. Estresores. Paradigma.

\begin{abstract}
The International Work Organization identifies stress as the first sign of a response to physical or emotional harm that could suppose loss between $0.5 \%$ and $3.5 \%$ of the Gross Domestic Product of each country. There is a paradigm within companies in which areas such as production and finances are considered to be more likely to present it, than their counterparts of human resources and marketing.
\end{abstract}




\section{Original}

ESTUDIO COMPARATIVO DE NIVELES DE ESTRÉS ENTRE ÁREAS FUNCIONALES DE EMPRESAS FAMILIARES. EL PARADIGMA DE ÁREAS DURAS Y BLANDAS.

OBJECTIVE: To identify if there is a difference in stress levels among those areas in the selected study units.

MATERIAL AND METHOD: A transactional descriptive research was made applying the Aldebarán-Test to identify the labor stress level, using a subject type sample in 35 medium family businesses located in Valle de Mexico. There were 280 participants in the sample which was integrated by Managers and Heads of the production, human resources, finance and marketing areas.

RESULTS: Stress levels were very similar in the four selected areas and the highest incidence was observed in the organizational factors.

CONCLUSIONS: The obtained findings provide information that help both, to identify concomitant factors related to stress levels and to design specific strategies to control them.

KEYWORD: Work stress. Family enterprises. Stressors. Paradigm.

\section{INTRODUCCIÓN}

Esta investigación, tiene como finalidad identificar si existe diferencia entre el nivel de estrés existente en cuatro áreas funcionales de empresas familiares, toda vez que, existe un paradigma que menciona que las áreas de producción y finanzas presentan un nivel superior de estrés con referencia a las áreas de recursos humanos y mercadotecnia. Se conceptualiza paradigma como, la determinación de creencias compartidas por la comunidad, (Kuhn, 2013); "tradicionalmente" se ha asignado a las áreas de producción y finanzas el título de "áreas duras" y a recursos humanos y mercadotecnia (donde para este trabajo se incluye el área de ventas) el título de "áreas blandas", tratando de indicar con ello que son áreas "más fáciles" y, por ende, de menos estrés.

Para ello, es importante mencionar que el estrés es la respuesta, tanto física como emocional a un daño ocasionado por un desequilibrio entre las exigencias percibidas y los recursos y capacidades percibidos de un individuo para hacer frente a esas exigencias (Organización Internacional del Trabajo, 2016). El estrés se encuentra relacionado con el trabajo y está determinado por la organización del trabajo, el diseño del trabajo y las relaciones laborales; se 
ESTUDIO COMPARATIVO DE NIVELES DE ESTRÉS ENTRE ÁREAS FUNCIONALES DE EMPRESAS FAMILIARES. EL PARADIGMA DE ÁREAS DURAS Y BLANDAS.

presenta cuando las exigencias del trabajo no tienen correspondencia o se exceden de las capacidades, recursos o necesidades del trabajador o cuando el conocimiento y las habilidades de un trabajador o de un grupo para enfrentar dichas exigencias no coinciden con las expectativas de la cultura organizativa de la empresa. Para la American Psychological Association (2018) es una "experiencia emocional molesta que venga acompañada de cambios bioquímicos, fisiológicos y conductuales predecibles". Por lo anterior, es factible considerar al estrés como un desequilibrio del organismo con manifestaciones fisiológicas, psicológicas y bioquímicas. Si bien, el estrés resulta ser algo cotidiano y en cierta forma podría ser considerado como algo normal que permite a la persona mantenerse alerta, en caso de llegar a ser crónico o excesivo puede tener consecuencias en la salud, afectando el sistema cardiovascular, neuroendocrino, inmunitario y nervioso, anexo a lo anterior, en el momento en que las demandas del trabajo resultan ser altas y los recursos con que se cuentan son limitados, los trabajadores enfrentan un desgaste energético y una desmotivación que favorecería el que se presente un desgaste profesional sin distinción de profesión.

Existen variables como las mencionadas por Edwards y Cooper (Padilla Montemayor, Peña Moreno, \& Arriaga González, 2006) en el modelo de adecuación persona-medioambiente, donde se sugiere que el daño ocasionado por el estrés resulta de la falta de integración entre los requerimientos y demandas del trabajo con respecto a la habilidad real o percibida de la persona para satisfacerlos. En este sentido, las diferencias individuales referentes a las percepciones, habilidades, tolerancia a la presión del trabajo y vulnerabilidad a los resultados no deseados son los modificadores claves de la relación estrés-daño, de tal forma, que es posible vislumbrar que existen factores organizacionales, extra organizacionales y físicos que influyen más en el nivel de estrés.

La importancia de conocer este tema, radica en que en las últimas décadas se ha presentado un aumento el estrés relacionado con el trabajo y por ende sus consecuencias, como son:

- Impacto en la salud: El estrés elevado contribuye a trastornos mentales y de comportamiento, como, el agotamiento, síndrome de burnout, desgaste, ansiedad, depresión, abuso de alcohol y drogas, aumento en el índice de accidentes laborales, enfermedad cardiovascular y trastornos musculoesqueléticos (Organización Internacional del Trabajo, 2016). 


\section{Original}

ESTUDIO COMPARATIVO DE NIVELES DE ESTRÉS ENTRE ÁREAS FUNCIONALES DE EMPRESAS FAMILIARES. EL PARADIGMA DE ÁREAS DURAS Y BLANDAS.

- Impacto económico: El estrés genera altos costos económicos tanto para la persona enferma como para sus familiares, ya que a menudo reducen su productividad tanto en el hogar como en el trabajo, pierden ingresos y además incurren en gastos de salud que puede agravar su economía, generando mayor estrés. La Organización Internacional del Trabajo (OIT), menciona que el estrés laboral podría suponer pérdidas de entre el 0.5\% y el 3.5\% del Producto Interno Bruto (PIB) de cada país (Rojas, 2017).

La importancia de realizar este estudio en empresas de tipo familiar se debe a que se considera que en el tejido empresarial mexicano el $90 \%$ de las empresas son precisamente de tipo familiar generando en derredor del $70 \%$ del empleo y el $60 \%$ del PIB. Se considera a las empresas familiares como "La unidad económica en la cual se pueda identificar claramente la participación de una o más familias que posean el control económico y/o participen en la administración y toma de decisiones de la organización" (Martínez González, Martínez García, \& Juárez García, 2018).

Hasta donde los autores conocen, no se han realizado estudios comparativos para comprobar lo que la "sabiduría popular" ha indicado hasta el momento, por lo que el objetivo de la investigación es identificar si existe diferencia en los niveles de estrés entre dichas áreas.

\section{OBJETIVO}

Identificar si existe diferencia en los niveles de estrés entre dichas áreas en las unidades de estudio seleccionadas.

Los factores considerados son:

- Estresores del ambiente físico.

- Estresores organizacionales.

- Estresores extra organizacionales.

- Nivel de estrés.

\section{MATERIAL Y MÉTODO}

Se realiza una investigación de tipo descriptiva, transeccional.

En la presentación de la encuesta se especificó: 
ESTUDIO COMPARATIVO DE NIVELES DE ESTRÉS ENTRE ÁREAS FUNCIONALES DE EMPRESAS FAMILIARES. EL PARADIGMA DE ÁREAS DURAS Y BLANDAS.

a) El objetivo del estudio.

b) Su naturaleza anónima (no se recogieron datos personales como nombre o algún dato que permitirá su identificación).

c) Que la información sería tratada en forma confidencial y analizada en conjunto para publicaciones científicas y de difusión en congresos especializados.

d) Que en ningún caso se publicarían datos individuales.

No existe conflicto de interés en la realización de este estudio.

Se utilizó el Aldebarán-Test (García-Valerio, 2003) para identificar los factores principales de generación de estrés laboral, este instrumento cuenta con criterios de validez y un Alpha de 0.7933, el cual está constituido por tres dimensiones, las cuales son:

- Estresores del ambiente físico.

- Estresores organizacionales.

- Estresores extra organizacionales.

Los cuales en su conjunto permiten obtener el nivel de estrés.

Los criterios de inclusión considerados para las personas fueron:

a) Que laboren en una empresa familiar clasificadas como medianas;

b) Tengan cargo gerencial o jefatura en áreas de producción, finanzas, recursos humanos, mercadotecnia.

Los criterios de exclusión considerados fueron:

a) Que no laboraran en empresas familiares clasificadas como medianas;

b) Que no tuvieran cargos gerenciales o de jefaturas en las áreas de interés para el estudio;

c) Que no hubieran completado correctamente el test.

Se obtuvo una muestra de sujeto tipo con un total de 280 cuestionarios.

Conceptualización de las variables consideradas: 
ESTUDIO COMPARATIVO DE NIVELES DE ESTRÉS ENTRE ÁREAS FUNCIONALES DE EMPRESAS FAMILIARES. EL PARADIGMA DE ÁREAS DURAS Y BLANDAS.

- Factores ambientales: Son los factores relacionados con los agentes contaminantes que se encuentran en el ambiente de trabajo (ruido, iluminación, temperatura, aire contaminado, diseño de área laboral).

- Factores organizacionales: Se consideran factores como la calidad de vida en el trabajo y los conflictos relacionados con la empresa en sí (estilo de dirección, preocupaciones profesionales, horario y turnos de trabajo económicos, clima organizacional).

- Factores extra organizacionales: Se toman en consideración los sucesos ajenos a la organización y el tipo de conducta (dificultades familiares, competitividad).

\section{RESULTADOS}

En la figura 1, se aprecia cómo se integró el total de la muestra por 280 participantes. Es interesante observar que en el área de recursos humanos, predominan el género femenino con un $64 \%$, en tanto que en mercadotecnia disminuye a un 33\%, en finanzas constituyeron el $17 \%$ y en producción solo el $4 \%$.

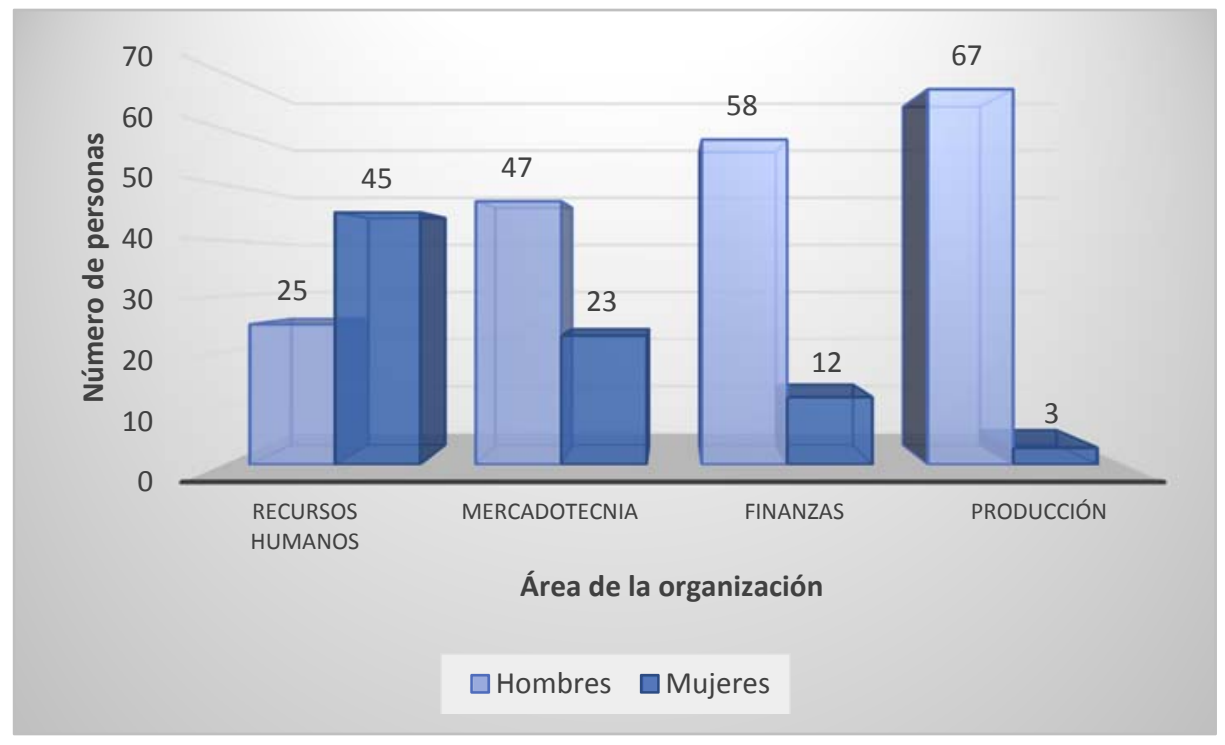

Figura 1. Total de la muestra.

Fuente: Elaboración propia.

En la figura 2, es posible realzar en porcentajes un comparativo de los niveles de estrés encontrados en los integrantes de la muestra. Estos se dividen dependiendo de los síntomas relacionados con el estrés, considerando tres niveles: 


\section{Original}

ESTUDIO COMPARATIVO DE NIVELES DE ESTRÉS ENTRE ÁREAS FUNCIONALES DE EMPRESAS FAMILIARES. EL PARADIGMA DE ÁREAS DURAS Y BLANDAS.

Leve: Es un nivel considerado como un estrés "cotidiano" en el cual no se presentan síntomas de afectación relacionados.

Moderado: Es el nivel de estrés donde se presentan síntomas de leves a moderados relacionados.

Grave: En este nivel se presentan múltiples y acusados síntomas relacionados.

Además, es factible observar, que si bien existen ligeras variaciones en lo relativo al nivel de estrés, éstas no dan un margen tan amplio como el que comúnmente se creería, encontrando que:

a) En el nivel considerado como grave, el área de producción que obtuvo el nivel más alto, su promedio es de un $47 \%$, en tanto que para recursos humanos es de un $40 \%$ y ésta brecha disminuye entre finanzas que presenta un 37\% y mercadotecnia con un $36 \%$.

b) En el nivel considerado moderado, el área de finanzas encabeza los resultados con un $50 \%$, seguido de mercadotecnia con un $47 \%$, muy cercano a recursos humanos con un $46 \%$ y próximo a producción con un $42 \%$.

c) En el nivel considerado como leve, el área de mercadotecnia muestra el mayor promedio con un $17 \%$, seguido de recursos humanos con un $14 \%$, muy cercano a finanzas con un $13 \%$ y producción con un $11 \%$.

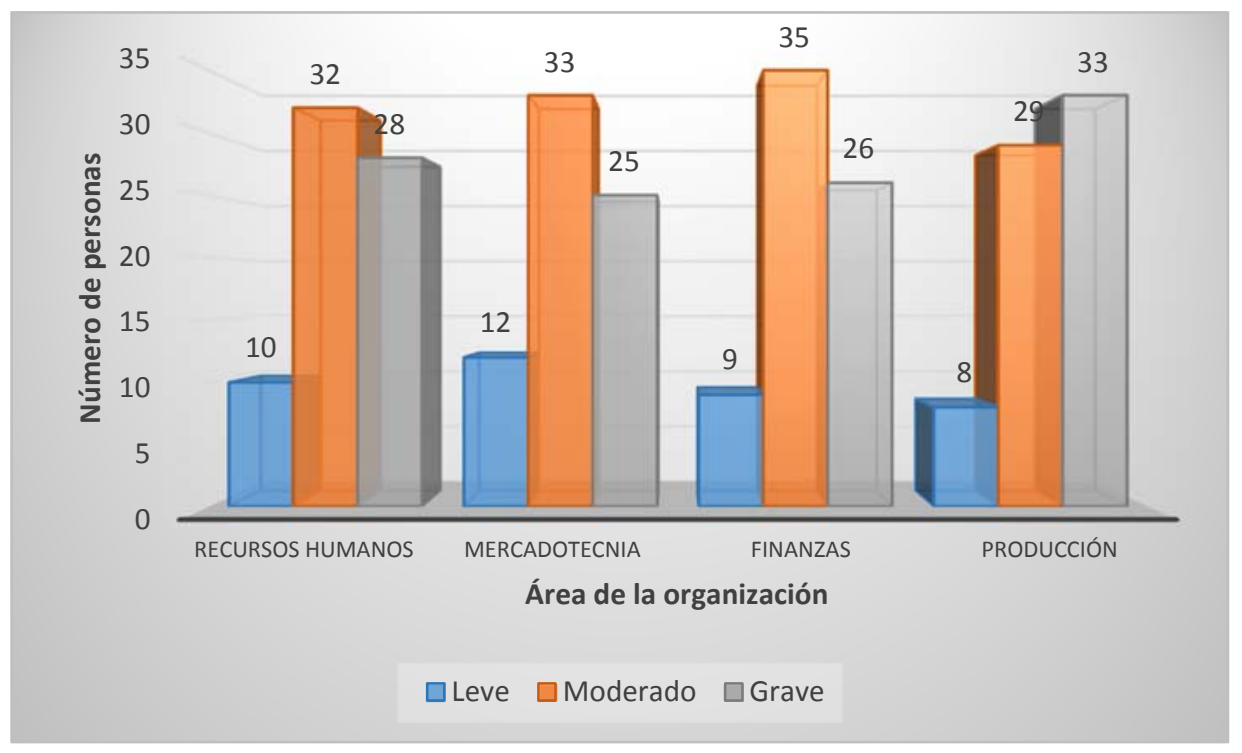

Figura 2. Niveles de estrés identificados en la muestra.

Fuente: Elaboración propia. 


\section{Original}

ESTUDIO COMPARATIVO DE NIVELES DE ESTRÉS ENTRE ÁREAS FUNCIONALES DE EMPRESAS FAMILIARES. EL PARADIGMA DE ÁREAS DURAS Y BLANDAS.

En la figura 3, se muestran en porcentajes los estresores referenciados por los integrantes de la muestra del tipo, encontrando que los estresores organizacionales predominan en forma importante, encontrando que:

a) En lo referente a los estresores del ambiente físico, el nivel más alto fue para producción con un $11 \%$, seguido por un empate entre recursos humanos y finanzas con un $6 \%$ y quien menos referenció verse estresado por este factor fue mercadotecnia con un $3 \%$.

b) En lo referente a los estresores organizacionales, el nivel más alto fue para producción con un $69 \%$, seguido por mercadotecnia con un $66 \%$, posteriormente finanzas con un $64 \%$ y por recursos humanos con un $61 \%$.

c) En lo referente a los estresores extra organizacionales, el nivel más alto fue para recursos humanos con un 33\%, seguido por mercadotecnia con un 31\%, finanzas con un $30 \%$ y finalmente producción con un $20 \%$.

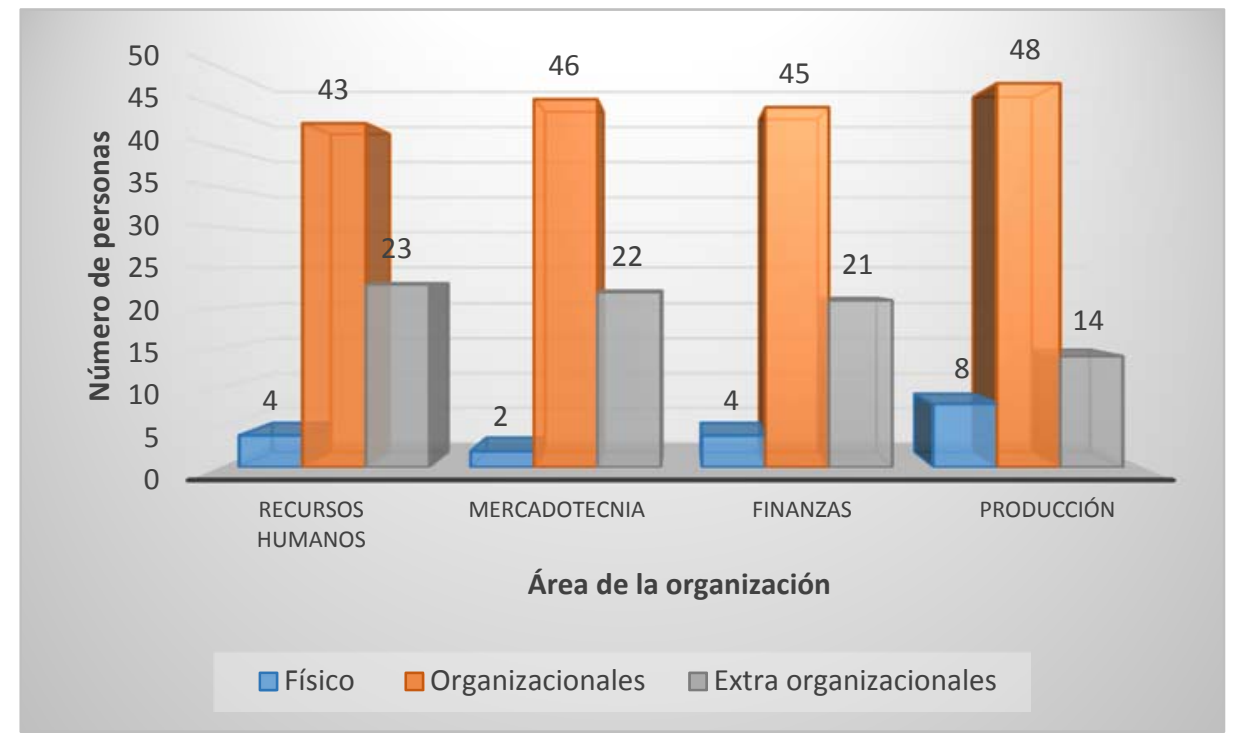

Figura 2. Factores de estrés identificados.

Fuente: Elaboración propia. 
ESTUDIO COMPARATIVO DE NIVELES DE ESTRÉS ENTRE ÁREAS FUNCIONALES DE EMPRESAS FAMILIARES. EL PARADIGMA DE ÁREAS DURAS Y BLANDAS.

\section{CONCLUSIONES}

Considerando los datos obtenidos en la muestra seleccionada, es factible concluir:

En lo relativo a los niveles de estrés, se pudo observar que el estrés moderado es el que se presenta en forma más recurrente con un 46.07\%, seguido muy de cerca por el estrés grave con un $40 \%$ y en nivel leve con un $13.93 \%$. Estos datos resultan relevantes, al tratarse de una muestra integrada por personal de nivel gerencial y de jefatura, brindando información que puede coadyuvar en la toma de decisiones para la realización de programas enfocados a mejorar la calidad de vida laboral de quienes ocupan este tipo de cargos.

En lo relativo a los tipos de estresores identificados, predominaron los organizacionales con un $65 \%$, en tanto que los extra organizacionales con un $28.57 \%$ y en los físicos un $6.43 \%$. En este sentido, es importante el resaltar que en esta muestra en particular los participantes mencionaron en forma reiterada que algunos de los principales estresores que encontraban en sus trabajos son:

a) Falta de procesos claros.

b) Condiciones subestándar para el desarrollo de los trabajos.

c) Procesos que son considerados "secretos" y son dominados por pocas personas.

d) Cadenas de mando poco claras y confusas.

e) Imposibilidad para implementar procesos más eficientes (existe una fuerte tendencia a emplear procesos que fueron útiles en años anteriores pero que ahora resultan poco eficientes o francamente obsoletos).

f) Sistemas de comunicación poco claros.

g) Sistemas de reclutamiento y selección ineficientes y/o atados a "restricciones" de criterios internos.

h) Urgencias constantes que no permiten desarrollar los programas acordados.

i) Un factor descrito como "juntitis"; es decir, que se invierte mucho tiempo del día en reuniones que resultan innecesarias. 


\section{Original}

ESTUDIO COMPARATIVO DE NIVELES DE ESTRÉS ENTRE ÁREAS FUNCIONALES DE EMPRESAS FAMILIARES. EL PARADIGMA DE ÁREAS DURAS Y BLANDAS.

Estos hallazgos brindan información valiosa, que puede coadyuvar tanto a identificar factores concomitantes en lo relativo a los niveles de estrés como al diseño de estrategias específicas para su control.

Considerando los hallazgos de la investigación, es factible mencionar que para esta muestra en particular:

1. Sí existe diferencia en los niveles de estrés de las áreas en las unidades de estudio seleccionadas; sin embargo, no resultan tan abismales como tradicionalmente se considera en el ámbito empresarial, ya que, si bien, en el nivel considerado como grave, producción presentó un $47 \%$, recursos humanos presentó un muy cercano $40 \%$ y entre finanzas (37\%) y mercadotecnia (36\%) la diferencia es de tan solo un 1\%. Lo anterior, resulta relevante, ya que brinda información que permite vislumbrar la importancia de considerar tanto a los integrantes de todas las áreas funcionales de la organización en los programas orientados al control y manejo del estrés.

2. Es posible observar, que los principales estresores identificados con la muestra seleccionada están generados por las propias empresas donde trabajan, siendo estos una fuente de áreas de oportunidad, que de ser identificados y subsanados por los líderes de las organizaciones, es factible pensar que lograrán ventajas significativas.

\section{REFERENCIAS BIBLIOGRÁFICAS}

American Psychological Association. (2018). Comprendiendo el estrés crónico. Recuperado de https://www.apa.org/centrodeapoyo/estres-cronico.aspx

Edwards, J. R., Caplan, R. D. y Harrison, R. V. (1998). Theories of organizational stress. Oxford: Oxford University Press.

García, A. (2003). Discriminación de factores de estrés laboral entre ejecutivos y personal operativo en una micro empresa del Valle de México (Tesis de maestría) Secretaría del Trabajo y Previsión Social. México.

Greenspan, F. (1993). Endocrinología básica y clínica. México: El manual moderno. 


\section{Original}

ESTUDIO COMPARATIVO DE NIVELES DE ESTRÉS ENTRE ÁREAS FUNCIONALES DE EMPRESAS FAMILIARES. EL PARADIGMA DE ÁREAS DURAS Y BLANDAS.

Instituto Mexicano del Seguro Social. (2018). Estrés Laboral. Recuperado de http://www.imss.gob.mx/salud-en-linea/estres-laboral

Iranzo Amatriain, J. M. y Blanco Merlo, J. R. (1999). Sociología del conocimiento científico. Madrid: Centro de Investigaciones Sociológicas (CIS).

Kuhn, T. S. (2013). La estructura de las revoluciones científicas. México: Fondo de Cultura Económica.

Lakatos, I. (1978). La metodología de los programas de investigación científica. Madrid: Alianza.

Martínez González, J. V., Martínez García, V. A. y Juárez García, A. C. (2018). Empresas familiares. Del abismo a la clase mundial. México: COLPARMEX, A.C.

Mayo Clinic. (2018). Síntomas de estrés: consecuencias en tu cuerpo y en tu conducta.

Recuperado de https://www.mayoclinic.org/es-es/healthy-lifestyle/stress-management/indepth/stress-symptoms/art-20050987

Mayo Castro, A., González Hernández, A. y Pérez Sánchez, B. (2016). Empresas familiares en México: Gobierno. Universidad Autónoma Nuevo León, 2(1), 2489-2512.

Organización Internacional del Trabajo. (2016). Estrés en el trabajo. Ginebra. Recuperado de https://www.ilo.org/public/libdoc/ilo/2016/490658.pdf

Organización Panamericana de la Salud. (2008). CIE-10 Clasificación estadística internacional de enfermedades y problemas relacionados con la salud (1). Washington, D.C.

Padilla Montemayor, V. M., Peña Moreno, J. A. y Arriaga González, A. (2006). Patrones de personalidad tipo A o B, estrés laboral y correlatos psicofisiológicos. Psicologia y Salud, 16(1), 79-85.

Page Personnel. (2017). México. Estudio de Remuneración. México: PageGroup.

Papalia, D. y Wendkos Olds, S. (1988). Psicología. México: McGraw-Hill.

Pichot, P., López-Ibor Aliño, J. J. y Valdés Miyar, M. (1995). DSM-IV Manual diagnóstico y estadístico de los trastornos mentales. Recuperado de http://www.mdp.edu.ar/psicologia/psico/cendoc/archivos/Dsm-IV.Castellano.1995.pdf

Rodríguez Carvajal, R. y De Rivas Hermosilla, S. (2011). Los procesos de estrés laboral y desgaste profesional (burnout): diferenciación, actualización y líneas de intervención. Med Segur Trab, 57(1), 72-88. 


\section{Original}

ESTUDIO COMPARATIVO DE NIVELES DE ESTRÉS ENTRE ÁREAS FUNCIONALES DE EMPRESAS FAMILIARES. EL PARADIGMA DE ÁREAS DURAS Y BLANDAS.

Rojas, R. (09 de abril de 2017). México continúa en primer lugar a nivel mundial en estrés laboral: OMS. Recuperado de https://saludiario.com/mexico-continua-en-primer-lugar-anivel-mundial-en-estres-laboral-oms/

Sociedad Española para el Estudio de la Ansiedad y el Estrés - SEAS. (2015). Depresión en el ámbito laboral - Informe de la World Federation for Mental Health. Recuperado de http://www.ansiedadyestres.org/content/depresi\%C3\%B3n-en-el-\%C3\%A1mbito-laboral\%E2\%80\%93-informe-de-la-world-federation-mental-health 